\title{
Many Worlds: Quantum Theory and Reality?
}

\section{IÑAKI SAN PEDRO ${ }^{1}$}

\section{Introduction}

Recent years have seen a good deal of work done on one of the most mind blowing and conceptually radical views in quantum mechanics, namely the 'many worlds' interpretation of quantum mechanics. Many worlds was first proposed by Hugh Everett III in his long PhD dissertation, which only some years later and after significative shortening came to be published as the 'Relative State Formulation of Quantum Mechanics' (Everett, 1957).

To commemorate the 50th anniversary of the first publication of Everett's ideas two conferences were held at Oxford University and the Perimeter Institute, which brought together some of the most active researchers in the field. The works presented in these two conferences have later been compiled in the volume 'Many Worlds? Everett, Quantum Theory and Reality', edited by Simon Saunders, Jonathan Barrett, Adrian Kent and David Wallace'2. This is then a comprehensive collection of papers - 23 papers altogether-, where the reader will find virtually anything that can be said about the many worlds interpretation today.

The book is organised in six parts, each addressing different though related questions of diverse interest and scope. These range from the ontological commitments of Everett's proposal (in the two first parts) - which stand in support of many worlds as offering a realist conception of quantum phenomena-, to questions regarding the role and 'place' of probability in the fully deterministic account that Everettian quantum mechanics conveys (in parts 3 and 4). These first four chapters put quite a lot of emphasis as well on the role that decoherence theory has taken in recent years. Decoherence, in fact, takes a central role in the book in as much as defenders of Everettian quantum mechanics see it as providing a solution both to problems related to the ontological view depicted by many worlds, as well as those related to the need to provide an adequate account of the probabilistic character of empirical observations of quantum phenomena. The reader will also find chapters - as those in part 5- discussing different alternative views to Everettian quantum mechanics, and other more historical works, in part 6, together with papers on the relation of many worlds to other disciplines, such as cosmology, or others that leave us with several interesting open questions for future research. Each part has a final section with the transcripts of the corresponding discussion sessions that took place after each talk.

Realism is perhaps the common thread throughout the book, a somehow default position shared by both defenders and opponents of the Everettian picture. This is already made clear from the very beginning, in the introduction to the book - a very helpful, long and thorough introduction and summary by Simon Saunders. Thus, for instance, much of the efforts by defenders of Everett's quantum mechanics are put, on the one hand, into making good sense of the ontological view that the theory purports. On the other hand, they also need to provide a sensible account of how probabilities enter the whole picture. By contrast, authors rejecting the many worlds view will stress the fact that Everettians are not able to give a proper and accurate ontological account of what these (so many) worlds are actually made of, nor a good story of how a fully deterministic theory can make sense of the probabilistic character of observed quantum phenomena.

The above is very rough characterisation of what the most central problems discussed in the book are. I shall discuss such issues a little bit more in detail in what follows, although I shall concentrate particularly on the problems Everettians face in relation to probability. Before proceeding, however, I would like to make some brief remarks on some of the questions that arise as regards the ontology of the Everett interpretation, which I found of great interest as well.

\section{Ontology, Decoherence and Emergent Structures}

Discussions on the ontological commitments, implications and difficulties of Everettian quantum mechanics can be found in the first two parts of the book. In brief, Everettians maintain that our world - the world we inhabit, that is - as described by quantum mechanics is consistent with many other worlds which, as wells as ours, are constantly branching in time. This is a consequence of our applying the formalism of quantum mechanics with no restriction to the whole universe. In particular, this is the picture that follows, it is claimed, if the Schrödinger equation is applied

\footnotetext{
${ }^{1}$ I would like to thank the members of the MCISR reading group, at Complutense University Madrid, who took the time and patience to go through several chapters of the book. They turned the weekly readings in helpful discussions and provided many useful comments.

${ }^{2}$ S. Saunders, J. Barrett, A. Kent, and D. Wallace (Eds.), Many Worlds? Everett, Quantum Theory, and Reality, Oxford University Press, 2010, 640 pages, hardback.
} 
to any macroscopic system. The quantum wavefunction therefore - which is taken to be something physically real in the Everett picture - is all we have, i.e. ontologically speaking, is all there is. The many continuously branching worlds then all constitute reality, i.e. they are all as real as our own world is.

The Everettian picture as described above, however, has an obvious problem. Namely, if the quantum wavefunction is all that there is, if we only have the quantum formalism, as given by the Schrödinger equation, how can we make sense of the experimental data observed (not just numerically but also ontologically)? More precisely, what is the actual 'stuff' in the Everettian picture that is capable of taking us from the very formalism to the empirical (macroscopic) observations? Can the wavefunction just by itself provide that 'connection', so to speak? This worry is raised by Tim Maudlin in his chapter 'Can the World Be Only Wavefunction?'.

The answer to the above questions, in Maudlin's view, is negative. What Everettians face, really, is an epistemic problem, not exclusive of many worlds but common to all so-called 'monistic' interpretations of quantum mechanics, such as GRW and other collapse interpretations. Maudlin's point, more precisely, is that it is not even logically possible for such monistic views to be able to fill in the gap between their theoretical machinery and the empirical phenomena these are supposed to account for. This is because the quantum formalism by itself — be it given by the Schrödinger equation with no further modification, be it a further version of it modified to accommodate collapse (as it is the case of GRW) — does not contain the right ontological 'stuff', i.e. actual particles. The quantum formalism, in other words, if taken just on its own, lacks the capacity to make the connection with the empirical data observed macroscopically. Only Bohmian mechanics can sensibly do that, Maudlin's argument goes. For it explicitly incorporates extra ontology on the top of the formalism.

By contrast, proponents of many worlds claim that it is decoherence that can do the job. This is the position defended in the three first papers of the book, by David Wallace, Jim Hartle, and Jonathan Halliwell. The appeal to decoherence theory is not free of difficulties, however. On the one hand, decoherence is elusive in some cases and even completely absent in some others (although this seems not to be a serious worry). On the other hand, decoherence theory is put at work in a context of 'emergence'. In that view, as defended by Wallace, 'emergent structures' correspond to the observed macroscopic objects. Again, this seems not enough for Maudlin. For in what sense, he insists, can be said that such structures emerge, if the theory does not contain an underlying fundamental ontology to start with? To this question, defenders of many worlds do not seem to have a good answer.

\section{Probability in Many Worlds}

One of the (perhaps most striking) features of the many worlds interpretation is that it is fully deterministic. In the first place, on branching (measurement), all possible outcomes are actually realized. On the other hand, the corresponding in-branch evolution is fully deterministic, for it follows the standard quantum mechanical unitary evolution given by the Schrödinger equation. Determinism, however, seems to be at odds with quantum mechanics, whose empirical observations have a strong stochastic character.

One of the most fundamental problems Everettians face is then, put very roughly, how to make sense of the probabilistic observations associated to quantum phenomena in a fully deterministic theory (such as many worlds). To my mind, this is perhaps the most central problem discussed in the book. For failing to give an appropriate account of how probabilities enter the many worlds picture, Everettians fail to explain not only observed empirical data but also to predict any experimental outcomes whatsoever. Most remarkably, Everettians will fail as well to be able to confirm (or disconfirm) their very theory by appealing to empirical observations, unless they can make sense of them in an appropriate way. This is the so-called 'problem of evidence'.

In order to solve the problem above, Everettians need to be able to give an account of branching amplitudes as probabilities (or at least to be able to relate them appropriately). But not any kind of probability. For recall that Everettians are committed to realism about the quantum state. Thus, whatever probabilities involved in the theory will need to be objective probabilities, or chances.

Simon Saunders, in his chapter 'Chance in the Everett interpretation' defends the view that, indeed, branching amplitudes in Everettian quantum mechanics are the equivalent to chances, i.e. objective probabilities, in one-world theories. To reach this conclusion, Saunders' efforts concentrate in showing that the specific features that characterise both branching amplitudes and chances are basically the same. In other words, branching amplitudes play the same role, we are told, in Everettian quantum mechanics as chances do in one-world theories. These chance roles, more specifically, are identified by Saunders to be: that they can be measured by statistics, they are related (numerically) to subjective degrees of belief, and they involve uncertainty. That branching amplitudes satisfy all 
these three chance-roles, is thus enough, in Saunders' view to say that the branching structures of Everettian quantum mechanics 'just are chances, or physical probabilities' (p. 182). What else can they be?

However, Saunders' argument, suggestive as it may be, does not constitute more than a qualitative argument in support of the chancy character of branching amplitudes. After all, there is nothing in the Everett formulation that explicitly identifies branching amplitudes with chances, so one should expect that no formal proof of such an equivalence be easily produced. Saunders is actually aware of this fact, and perhaps precisely because of that he endorses the view that probabilities need not be fundamental. Indeed, the correspondence between branching amplitudes and chances will make sense as long as probability is seen as an emergent feature of the theory rather than a fundamental one. As Saunders himself claims 'It is simply a mistake, if this reduction is successful, to see quantum theory as at bottom a theory of probability' (p. 182). (This, of course, is well inline with the fully deterministic character of the many worlds picture.)

Saunders arguments in 'Chance in the Everett interpretation' refer only, in any case, to two of the chance-roles above, i.e. the correspondence with statistics and the uncertainty they involve. As for the third (the second in his list), i.e. the link to subjective degrees of belief, Saunders points the reader to David Wallace's chapter 'How to Prove the Born Rule'. In this chapter, Wallace offers what I take to be one of the strongest results in the book. Namely, the (formal) derivation of the Born rule from decision theory considerations. In particular, Wallace proves a representation theorem by which, given a set of 'axioms of rationality' and a specific preference order, a utility function is derived such that the Born rule is the only possible probability distribution on the set of all possible worlds following a measurement operation.

As I said, the derivation of the Born rule is a strong result and, if valid, would considerably help Everettians to get their points through as regards the (apparent) clash of many worlds with the probabilistic character of quantum phenomena. Wallace's representation theorem is crucial, for instance, for arguments such as Saunders' above to go through. In fact Saunders' argument for the identification of branching amplitudes and objective probabilities hinges explicitly on a proper account of why the observed statistics are such and such, and how they are to be related to our degrees of belief. Also, for the defenders of the so-called 'fission programme', such as Hilary Greaves or Wayne Myrvold, some good argument to link chances with subjective degrees of belief is needed. (Only issues about uncertainty are dismissed by proponents of the 'fission programme'.)

Wallace's proposal however raises a number of worries, both as regards the actual plausibility of set of axioms of rationality assumed, as well as in relation to the implications of the result itself, i.e. of the representation theorem.

A first objection to Wallace would be to ask whether the axioms of rationality required for the derivation of the Born rule do indeed reflect rational action. Worries of this kind are expressed in the chapters by Huw Price and David Albert, for instance, who provide different arguments suggesting that there are good reasons to doubt that the axioms of rationality required by Wallace are in fact reasonable (rationality) constraints.

Price's argument goes even deeper in that it criticises the whole application of decision theory to the many-worlds framework. In Price's view, the standard decision theory approach, where a specific utility function - the Born rule, according to Wallace's representation theorem - is maximized, is valid only in a one-world picture. In a manyworlds context, on the other hand, standard decision theory cannot be applied. For agents in a many-worlds context will simply have different preferences than those in one-world. They should take into account, for instance, the fact that there will be many future selves - all of whom they should want to worry about - rather than just oneself. Alternatives to the Born rule are then available and are by no means 'irrational'. Price gives an example of such alternative rule, taken from political philosophy. Namely, the so-called 'distributive justice' rule.

From a somehow different point of view David Albert shows how capricious the choice preference order (needed for the proof of the representation theorem) can be. He suggests that almost any other choice, rather than the Born rule, would do. Albert provides an example, namely what he calls the 'fatness rule', under which Everettian branching amplitudes would correspond to a branching dynamics based on the actual fatness of the subjects in each branch. (Since fatness is directly associated to mass, the fatter a subject is the larger the corresponding branch amplitude must be.) The point is that no matter how awkward the rule is, it needs not be 'irrational'. Thus, there is no good justification for the specific preference order Wallace choses in his derivation of the Born rule.

Wallace is of course aware of these problems and he does a good job in trying to identify which of the axioms and assumptions that come in the derivation of his quantum representation theorem fail in each case. (He offers at the final part of his chapter a survey of the possible ways the quantum representation theorem can be criticised and how each of these is related to the specific violation of one or more of his axioms of rationality.) For Wallace therefore both Albert's 'fatness rule' and Price's 'distributive justice' critiques are unsound because they violate specific 
constraints of rationality. (Both Albert and Price would be seen as acting 'irrationally' in that sense. But this is exactly the point they want to make: their choices need not be irrational, they just do not maximize a certain expected utility).

Finally, even if the problems above can be dealt with and overcome, Everettians will still need to make sense of Wallace's derived probability measure as giving raise to proper chances. (As pointed out, this is absolutely crucial for arguments such as Saunders' sketched above to go through.) For what Wallace's representation theorem shows -always setting aside the problems above and taking for granted that the axioms of rationality assumed are plausible and rational indeed - is just that the Born rule is the only possible probability distribution that can be attached to the branching amplitudes in Everettian quantum mechanics. Thus, Wallace's representation theorem would be of no use if we would not be able to say that these branching amplitudes are in fact objective probabilities and not just the product of our subjective (even if rational) judgement.

Everettians, it would seem, could try to find what they need in the 'principal principle'. For, it is the 'principal principle' what, as it is usually taken, can get us from subjective degrees of belief to chances, i.e. what provides the link between them two.

Appealing to the 'principal principle' raises further worries, though. First, the 'principal principle' only ensures that subjective degrees of belief and chances are quantitatively linked. In other words, the 'principal principle' does not provide any robust link between chances and subjective degrees of belief, other than a qualitative correspondence. But this seems not to be enough, if we speak of chances, or objective ('physical') probabilities, i.e. there is more to chances, it seems, than just their (numerical) quantity. Second, recall that what the 'principal principle' says, exactly is that 'all else being equal, one who believe that the chance of $E$ is $p$ will set his credence in $E$ equal to $p^{\prime}$ ' (p. 181). Agreed, if we take the 'principal principle' to hold - and even we are able to draw some conclusions about what it is constitutive of chances, apart from their quantitative value - we seem to be allowed to set our credences by looking at chances. But can we also do the other way around? That is, can we set chances by looking credences all the same? I don't think so. And if we cannot do that then, again, Wallace's representation theorem — or any other result involving decision theory, explicitly derived using subjective probabilities - does not seem to be quite what we need if we are to provide a realist picture of quantum phenomena. Everettians are well aware of such difficulties. But also proponents of alternative one-world views, they claim, will need to worry about such issues. In fact, as pointed out by Saunders in the introduction to the book, no one-world alternative fares actually better than Everettian quantum mechanics when it comes to explain how the 'principal principle' may provide a robust link between chances and subjective degrees of belief. Fair enough, then, many worlds seems to be on a par with other one-world views. But is this really a good argument to favour one option over the other? Once more, I should say no. For the problem still remains.

\section{Open questions and final remarks}

I would like to conclude with some brief remarks and open questions about how may causation fit into the many worlds picture of quantum mechanics.

As in other realist proposals, many worlds should perhaps be expected to say something about causation. None of the papers in the book however tackle the issue per se. Bu it should have been interesting to have found some amount of discussion as to how, or in what sense, causal processes or relations may be found or characterised in the Everett interpretation. In particular, we may want to ask to what extent Everettian quantum mechanics can accommodate causal talk. And if so, where exactly in the formulation of the theory such causal talk should be present. There is no space here to address such questions in detail. But some brief remarks may do.

An obvious first reaction to questions as the above would be, of course, to note that since many worlds is a fully deterministic theory - recall again that all possible outcomes are actually realized, and the in-branch dynamics is fully deterministic - , any considerations about causality may well be described as in any other deterministic theory, such as for instance Newtonian mechanics.

Thus, if we pay attention to the in-branch (deterministic) dynamics, it would seem, effects inevitably (deterministically) follow their causes. But looking at the in-branch dynamics in more detail, do claims about causality really make sense? Everettians may actually be facing a similar problem here than they had with ontology. Namely, given that the in-branch dynamics is fully specified by the time (unitary) evolution as prescribed by the Schrödinger equation, one can require them to explain in what such unitary dynamics responds to some causal structure. Put it another way, since the Schrödinger equation provides a description of the time evolution of 
undisturbed quantum states, what is causal about it? Unitary evolution, again, seems to lack the appropriate 'stuff' (again at a fundamental ontological level) to make sense of such things as causes, effects, or causal relations.

As an alternatively, some idea of causation - either characterised by the actual causal relata themselves or just by causal relations - may well be thought to be present not at the level of the particular branches but rather at the level of branching itself. After all, if we take it that causation has to do with (physical) interactions rather than anything else, measurement interactions, and therefore branching, would seem to be playing a crucial role. In this view then, what would the Everettian picture entail?

Recall, once more, that one of the features of many worlds is that, on branching, all possibilities, i.e. all possible outcomes, are realized, meaning that branching itself is fully deterministic process. Now if we take branching to be constitutive of some sort of causal process, as suggested above, we need to conclude then that a cause in Everettian quantum mechanics has inevitably a variety of effects, all of which are fully determined by it. The problem with this view, again, is that it seems difficult to be able to explain why such effects (outcomes) indeed follow the Born rule,. Why, in other words, if all the effects are equally (deterministically) caused, those that belong to 'high amplitude' branches occur considerably more often than those belonging to branches with 'low amplitude'?

Again, this is not the place to assess such issues but, as I said, it would have been interesting to find a paper in the book addressing them in some detail.

To conclude then, after a reading of 'Many Worlds? Everett, Quantum Theory and Reality', one realises that Everettian quantum mechanics is a hot topic both in foundations as well as in philosophy of quantum mechanics. A proof of that is the wide interest that the two conferences at Oxford and the Perimeter Institute attracted among first figures in the field. (Thus the impressive line-up of contributors to the volume.)

Thus, despite the numerous and important problems it faces, we can say that many worlds is very much alive as one of the possible ways quantum mechanical phenomena can be made sense of. Its proponents will urge us, of course, to pay more attention to what Everettian quantum mechanics still has to say, never mind how radical, controversial or strange it may be - never was quantum mechanics a conceptually clear subject, anyway. Its opponents, on the other hand, may claim that there is a lot, too much perhaps, to make sense of in the theory for it to achieve what it claims it does, i.e. a realistic account of quantum phenomena.

For us all, in any case, it remains to be seen whether, or how, after further development in the foundations of quantum mechanics, many worlds will survive as a sensible realist picture of quantum phenomena. And turn out to be, after all, one of those things our grandchildren should get to know.

Complutense University of Madrid

Ciudad Universitaria, 28040 Madrid, Spain

inaki.sanpedro@filos.ucm.es

\section{References}

Everett III, H. 1957. “"Relative state” formulation of quantum mechanics', Reviews of Modern Physics, 29: 454462, reprinted in DeWitt and Graham (1973), The Many-Worlds Interpretation of Quantum Mechanics, Princeton University Press.

Greaves, H. 2007. On the Everettian Epistemic Problem. Studies in History and Philosophy of Modern Physics 38:120-152

Saunders, S and, D. Wallace 2008. Branching and uncertainty. British Journal for the Philosophy of Science 59:293 $-305$ 